Miguel Ángel Barbas Monjo $0^{1, *}$

Jara Velasco García Cuevas ${ }^{2}$

Jesús Rodríguez Lastra ${ }^{3}$

Juan Nicolás Cuenca Zaldívar ${ }^{4}$

1. Diplomado en Enfermería. Máster en Ciencias de la Enfermería. Doctorando. Hospital Guadarrama. Madrid. España.

2. Médico Geriatra. Hospital Guadarrama. Madrid. España.

3. Profesor de Fisiología, Universidad de Carabobo, Valencia, Venezuela. Profesor asociado, Universidad Autónoma de Madrid, Madrid, España.

4. Doctorando, Graduado en fisioterapia, Hospital Guadarrama, Madrid, España. Profesor del Grado de Fisioterapia, Universidad Francisco de Vitoria, Madrid, España.

*Autor para correspondencia.

Correo electrónico: miguelangel.barbas@salud.madrid.org (Miguel Ángel Barbas Monjo).

\section{Radiofrecuencia en la cicatrización de heridas crónicas. Una revisión en hospital de media estancia}

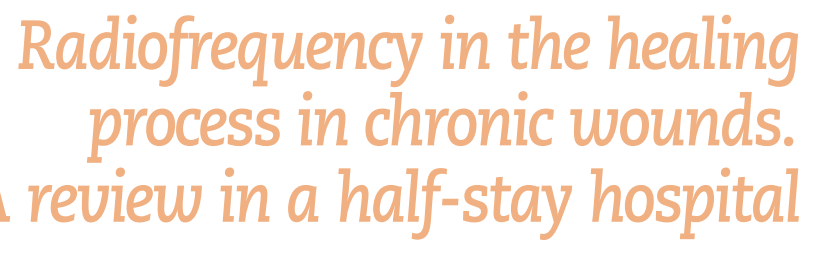

Recibido el 29 de octubre de 2019; aceptado el 19 de febrero de 2020.

\section{RESUMEN}

Las heridas crónicas son un problema de salud significativo. Parece que la estimulación eléctrica produce una reducción significativamente mayor en el área de superficie y cicatrización más completa de las úlceras de difícil cicatrización y de evolución tórpida en comparación con la terapia habitual, sin vendaje compresivo. Objetivos: Evaluar el efecto que la radiofrecuencia a baja intensidad y con efectos no térmicos tiene sobre los diferentes componentes del mecanismo del proceso de cicatrización. Metodología: Para el tratamiento, se utilizó un dispositivo de tecarterapia (CAPENERGY

C200). Se aplicaron un total de 10 sesiones de radiofrecuencia con una periodicidad de 1 vez a la semana con una potencia del $60 \%$ y una frecuencia de 1,2 MHz durante 30 minutos. Resultados: La presencia de edema, observada en todos los pacientes en la región de la extremidad inferior, desapareció en 30 de los 36 pacientes (Wilcoxon $p=0,004$ ). Este resultado fue confirmado por ultrasonido. El edema celular subcutáneo medio disminuyó en $1,73 \mathrm{~cm}$ (Friedman $p=0,000$ ). La temperatura del área tomada antes y después del tratamiento se incrementó en un promedio de $1,4^{\circ} \mathrm{C}$. Estas diferencias son estadísticamente significativas (Wilcoxon $\mathrm{p}=0,000$ ). Conclusiones: La radiofrecuencia parece que puede reducir el largo proceso de cicatrización de las lesiones de evolución tórpida, y nos encontramos con unas diferencias significativas a lo largo del tratamiento y con una reducción progresiva en las mediciones de las lesiones y mayor rapidez en la cicatrización de las heridas complejas.

PALABRAS CLAVE: Heridas crónicas, radiofrecuencia, pie diabético.

\section{ABSTRACT}

Chronic wounds are a significant health problem. Electrical stimulation seems to produce a significantly greater reduction in surface area and more complete healing of difficult-to-heal and poorly healing ulcers compared to standard therapy without compressive bandaging. Objectives: To evaluate the effect that radiofrequency at low intensity and with non-thermal effects has on the different components of the mechanism of the healing process. Methodology: A tecartherapy device (CAPENERGY C200) was used for the treatment. A total of 10 radiofrequency sessions were applied once a week with a power of $60 \%$ and a frequency of $1.2 \mathrm{MHz}$ for 30 minutes. Results: The presence of oedema, observed in all patients in the lower extremity region, disappeared in 30 of the 36 patients (Wilcoxon $\mathrm{p}=0.004$ ). This result was confirmed by ultrasound. The mean subcutaneous cellular oedema decreased by $1.73 \mathrm{~cm}$ (Friedman $\mathrm{p}=0.000$ ). The temperature of the area taken before and after treatment increased by an average of $1.4^{\circ} \mathrm{C}$. These differences are statistically significant (Wilcoxon $\mathrm{p}=0.000$ ). Conclusions: Radiofrequency appears to be able to reduce the long healing process of torpidly evolving lesions, and we found significant differences throughout the treatment and a progressive reduction in lesion measurements and faster healing of complex wounds.

KEYWORDS: Chronic wounds, Radiofrequency, Diabetic foot.

\section{У INTRODUCCIÓN}

Las heridas crónicas son un problema de salud significativo, especialmente en personas ancianas e individuos con lesión de la médula espinal $\mathrm{u}$ otras enfermedades que cursan con limitación de la movilidad, donde las lesiones por presión (LPP) hacen a la cronicidad. Las modalidades tradicionales de tratamiento de las LPP incluyen el uso de superficies de apoyo, cambios de posición, equilibrio nutricional, cambios frecuentes en el apósito, hidroterapia, desbridamiento y reconstrucción quirúrgicos. Las heridas abiertas crónicas a menudo requieren muchos meses de tratamiento $y$ reducen la calidad de vida de los pacientes ya enfermos ${ }^{1-3}$. El progreso generalmente es lento y, a menudo, incompleto. Con demasiada frecuencia, en pacientes con arteriopatía periférica diabética, la amputación de un miembro afectado se convierte en la única alternativa ${ }^{4}$.

Las lesiones complejas de la piel constituyen una patología que presenta una prevalencia creciente en este tipo de heridas, con el consecuente retraso en la cicatrización, que constituye un importante problema de salud pública ${ }^{5}$. La principal secuela es el retardo en el proceso cicatricial, como consecuencia de una disminución importante en la calidad de vida de estos pacientes y la de sus cuidadores ${ }^{1}$.

Estas lesiones también representan una enorme carga económico-financiera para el sistema de salud y para los pacientes. Pelham et al. ${ }^{6}$ 
señalaron que, aunque los costes específicos asociados con las LPP son difíciles de determinar debido a comorbilidades en individuos con enfermedades crónicas, el cuidado hospitalario de pacientes con LPP cuesta un $50 \%$ más que el cuidado de pacientes sin úlceras. Hirshberg et al. ${ }^{7}$ concluyen que el gasto hospitalario promedio es de 48934 dólares por paciente para el tratamiento de las úlceras, que no incluía el tratamiento antes de la hospitalización o el coste de los productos farmacéuticos. Otros estudios estiman los costes de tratamiento en miles de millones de dólares por año en los Estados Unidos ${ }^{8,9}$. Existe la necesidad de un tratamiento eficaz que disminuya el tiempo de cicatrización y la gravedad de las úlceras, sea fácil de usar y sea rentable.

La formación de la regeneración en el proceso de cicatrización de las heridas transcurre después de la finalización de la fase de remodelación; la cicatriz entra en el denominado estado maduro según el esquema propuesto por el Panel Consultivo de la International Advisory Panel on Scar Management ${ }^{10}$. La cicatriz no debe tener apéndices epidérmicos y mostrar un patrón de colágeno de fibras densamente empaquetadas. La resistencia a la retracción de la piel de la herida alcanza a la primera capa de piel o epidermis y no en la propia lesión ${ }^{11}$. Además, la cicatriz suele estar debilitada y con menos elasticidad que la piel normal ${ }^{12}$.

Los factores de crecimiento que estimulan las células necesarias para la reparación de tejidos se han identificado a nivel molecular. En las heridas crónicas, estas moléculas son deficientes, y se interrumpe la señalización de fibroblastos y células endoteliales (entre otras) para proliferar y comenzar el proceso de cicatrización ${ }^{13,14}$.

Las lesiones de extremidades inferiores (úlceras arteriales y venosas crónicas) se pueden considerar como procesos inflamatorios desregulados producidos por un suministro inadecuado de sangre, anoxia tisular, edema, muerte celular e infección, entre otros factores ${ }^{15}$. Estos cambios alteran la interacción entre los componentes estructurales de los tejidos afectados y entre estos y las células inmunes de una manera que impide la cicatrización de las heridas. Las hipótesis sobre la fisiopatología de las ulceraciones de miembros inferiores se centran en efectos locales inducidos por alteraciones hemodinámicas ${ }^{16-22}$. Hay tratamientos en la actualidad centrados en el alivio de estos cambios locales que incluyen medidas preventivas hemodinámicas, vendajes para úlceras, tratamientos tópicos y reparación quirúrgica o endovascular ${ }^{16,17}$. Los tratamientos experimentales exitosos incluyen transferencia génica intramuscular ${ }^{23}$, piel alogénica $\mathrm{Graft}^{24}$ y campos electromagnéticos directamente aplica$\operatorname{dos}(\mathrm{ELF})^{25,26}$.

Parece que la estimulación eléctrica produce una reducción significativamente mayor en el área de superficie y cicatrización más completa de las LPP de categoría II a IV en comparación con la terapia habitual, y en pacientes con arteriopatía periférica diabética según la clasificación de Meggitt-Wagner (grado 0 a 2) ${ }^{23}$. La eficacia de otros tratamientos complementarios, como la terapia electromagnética para mejorar el cierre completo de las LPP y promover las fases de curación de heridas, se ha utilizado en forma de microcorriente electromagnética $^{24}$. La radiofrecuencia comenzó a utilizarse en la práctica clínica al inicio de los años cincuenta. Sin embargo, en los últimos años, debido a la aparición de nuevos equipos y material, se ha conseguido disminuir los efectos secundarios, lo cual ha permitido que su empleo comenzara a generalizarse.

La regeneración de estructuras complejas sigue un proceso de morfostasis, definida como la forma en múltiples escalas de tamaños y niveles de organización en que un sistema biológico se adapta a las condiciones del entorno y gracias al recambio celular, lo que permite hacer frente al envejecimiento de los tejidos, a la remodelación y cicatrización de heridas y a las lesiones mayores que requieren regeneración. Los campos electromagnéticos parecen actuar facilitando estos procesos de regeneración a través de los mecanismos electrofisiológicos; esto se denomina potencial transepitelial (PTE). La cicatrización de heridas y la regeneración de tejidos son impulsadas por un sistema de autorreparación de circuito cerrado que utiliza señales (eléctricas) para iniciar la reparación después de una lesión ${ }^{27}$. Estas intervenciones biofísicas parecen modular los campos electromagnéticos endógenos alterados y ayudar en el restablecimiento de los procesos de cicatrización ${ }^{28}$. El ultrasonido y las terapias de presión y de luz han demostrado tener beneficios clínicos, y sus mecanismos moleculares parecen implicar alteraciones tanto biofísicas como bioquímicas.

\section{$\searrow$ OBJETIVOS}

El objetivo de este estudio es evaluar el efecto que la aplicación de radiofrecuencia a baja intensidad (frecuencia) y con efectos no térmicos tiene sobre los diferentes componentes del mecanismo del proceso de cicatrización de lesiones de difícil cicatrización.

\section{$\checkmark$ METODOLOGÍA}

En el estudio fueron incluidos 36 pacientes del Hospital de Guadarrama en Madrid (Espańa) que pasaron por la consulta durante el periodo de septiembre de 2018 a junio de 2019. (tabla 1). Trece hombres y $10 \mathrm{mu}$ jeres tenían diabetes mellitus.

Tabla 1. Características basales de la población de estudio

\begin{tabular}{|l|c|c|}
\hline & Mujer $(\mathbf{n = 2 1})$ & Hombre $(\mathbf{n = 1 5})$ \\
\hline Edad & $54,8 \pm 8,2$ & $72,6 \pm 7,5$ \\
\hline Altura & $160,5 \pm 6,8$ & $165,3 \pm 4,1$ \\
\hline Peso & $62,4 \pm 3,0$ & $78,6 \pm 4,1$ \\
\hline Diabetes mellitus & 10 & 13 \\
\hline PUSH & $14,4 \pm 2,2$ & $12,4 \pm 2,5$ \\
\hline Lesiones de extremidades inferiores. & $78,1 \pm 2,0$ & $21,9 \pm 2,4$ \\
\hline Vascular venosas & $11,7 \pm 2,1$ & $88,3 \pm 2,5$ \\
\hline $\begin{array}{l}\text { Lesiones de extremidades inferiores. } \\
\text { Vascular arteriales }\end{array}$ & & \\
\hline
\end{tabular}

Datos expresados con media \pm desviación típica.

PUSH: Pressure Ulcer Scale for Healing.

Criterios de inclusión: lesiones de larga evolución, difícil cicatrización, evolución tórpida, y que solo con el tratamiento convencional no ha finalizado el proceso.

Los participantes del estudio fueron debidamente informados sobre los objetivos del estudio y los posibles riesgos y beneficios. Todos ellos firmaron el formulario de consentimiento libre, previo e informado tan pronto como aceptaron participar en el estudio, cumpliendo así con la Declaración de Helsinki y según lo aprobado por el Comité de Ética en Investigación del Hospital Puerta de Hierro de Madrid (acta n. ${ }^{\circ}$ 02.18). Fue registrado en ClinicalTrials.gov (NTR: 03048799).

\section{Diseño}

Estudio unicéntrico, observacional analítico, prospectivo, de cohorte única dinámica con diseño de antes-después y medidas repetidas a lo largo del mismo. Está investigación tiene un enfoque clínico en el que la unidad de análisis serán las úlceras que presenten los pacientes tratados. 
Para poder participar en el estudio, los pacientes han cumplido los criterios de inclusión siguientes: a) Comprender y firmar voluntariamente los correspondientes consentimientos informados y hoja de información antes de que se realice ninguna evaluación/procedimiento relacionado con el estudio. b) ser hombre o mujer $\geq 18$ años $\mathrm{y} \leq 90$ ańos en el momento del consentimiento. $c$ ) Tener el diagnóstico de herida de larga evolución, difícil cicatrización y de evolución tórpida, que con tratamientos convencionales no ha llegado al final del proceso. d) Primera vez en el Hospital Guadarrama para recibir tratamiento.

Criterios de exclusión: $a$ ) portador de marcapasos o $b$ ) implantes metálicos locales.

\section{Intervención}

Para el tratamiento, se utilizó un dispositivo de tecarterapia C200 CAPENERGY Vascular (CE120). Las sesiones de radiofrecuencia se administraron con el aparato Tecarterapia C200 con sonda de pie modelo C-Boot, en el caso de lesiones en la planta del pie, o con placas capacitivas en el resto de las zonas corporales (fig. 1). Los parámetros dispuestos fueron una potencia del $60 \%$ y una frecuencia de $1,2 \mathrm{MHz}$ durante 30 minutos, que genera una sensación de calor que nunca supera el umbral de tolerancia del paciente.

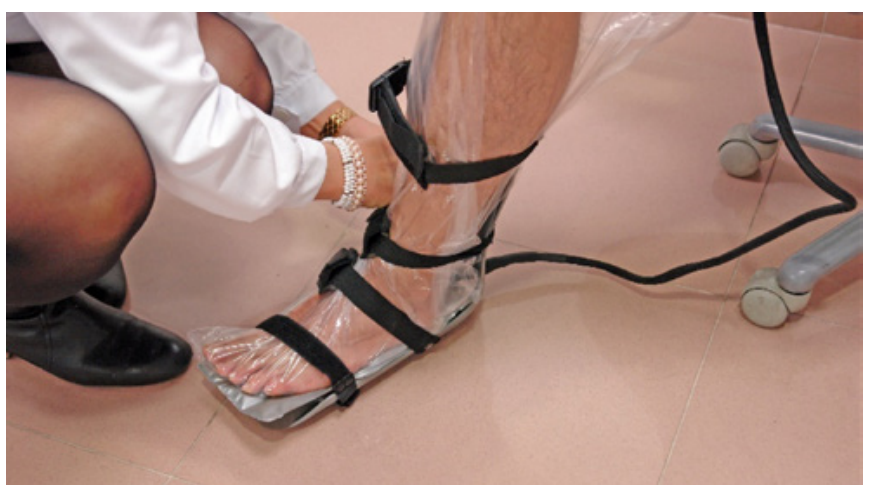

Figura 1. Dispositivo en forma de bota para usar con CAPENERGY C-200, para aplicar tratamiento de radiofrecuencia en el pie diabético.

Se aplicaron un total de 10 sesiones de radiofrecuencia con una periodicidad de 1 vez a la semana, con una potencia del $60 \%$ y una frecuencia de 1,2 $\mathrm{MHz}$ durante 30 minutos, colocándose sobre la lesión el cabezal de tratamiento y administrándose una dosis atérmica de hasta $37^{\circ} \mathrm{C}$.

\section{Variables del estudio}

Tras cada sesión de tratamiento se administró la escala Presure Ulcer Scale Healing (PUSH ${ }^{29}$. Esta escala consta de tres parámetros o subescalas: área de la herida, relacionado con la superficie de la lesión; longitud $x$ anchura (en dirección cefalocaudal y su perpendicular) en centímetros cuadrados; cantidad de exudado y apariencia local presente en la lesión, según el tipo de tejido en el lecho de la herida, pudiendo variar desde el tejido necrótico hasta el tejido epitelial o cicatrizado. Cada parámetro se puntúa según un sistema de puntuación, se suman las puntuaciones de la subescala y se obtiene una puntuación total del estado de la lesión. Las puntuaciones pueden oscilar entre 0 (cicatrizado) y 17 puntos (peor estado de la lesión posible).

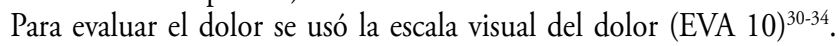
Dicha escala mide la intensidad del dolor y consiste en una línea hori- zontal de $10 \mathrm{~cm}$. En la izquierda se ubica la ausencia o menor intensidad y en la derecha la mayor intensidad. La valoración será: 1 = dolor leve si el paciente puntúa el dolor como menor de 3; 2 = dolor moderado si la valoración se sitúa entre 4 y 7 ; y 3 = dolor intenso si la valoración es igual o superior a 8 .

Como variable de la disminución y proporción de pacientes con edema al principio y al final del estudio, sin tratamiento de vendaje compresivo de multicomponentes.

\section{Análisis estadístico}

Para el análisis estadístico se utilizó el programa R (Versión 3.5.1). El nivel de significación se estableció en $p<0,05$.

La distribución de las variables cuantitativas, tanto basales como de resultado, se testó con la prueba de Shapiro-Wilk.

Las variables cualitativas se describieron en valores y frecuencias absolutos, y las variables cuantitativas con media y desviación típica o con mediana y rango intercuartílico (IQR) en función de presentar o no una distribución normal.

Para las variables cualitativas pre-postratamiento se utilizó el test de McNemar y de McNemar-Bowker en función de presentar una o más categorías.

En el caso de las variables cuantitativas repetidas a lo largo de las sesiones se utilizó la prueba de Friedman con test post hoc con corrección de Bonferroni; en caso de no encontrarse diferencias, se aplicaron correcciones menos estrictas: Holm, Benjamini \& Hochberg y Tukey. En caso de que la prueba de Friedman no mostrarse diferencias significativas, se aplicó un Anova robusto de medidas repetidas con medias truncadas al $20 \%$ y bootstrap.

El tamaño de efecto en las variables cuantitativas se definió, en las comparaciones pre-postratamiento, con la $\mathrm{D}$ de Cohen, definiéndose como 0,2 a 0,5 (pequeńo), 0,5 a 0,8 (moderado) y más de 0,8 (grande), y en las medidas repetidas como W de Kendall: menor de 0,10 (pequeño), 0,10 a 0,20 (mediano) y más de 0,20 (grande). La fuerza de asociación en las variables categóricas $\mathrm{G}$ de Cohen se definió como menos de 0,15 (pequeño), 0,15 a 0,25 (moderado) y más de 0,25 (grande).

\section{$\vee$ RESULTADOS}

La presencia de edema, observada en todos los pacientes en la región de la extremidad inferior, desapareció en 29 de los 36 pacientes; tratamiento habitual sin vendaje compresivo. Se encontraron diferencias significativas a lo largo del tratamiento $\left[\chi^{2}(2)=62,242, p<0,001\right]$ y un tamańo de efecto grande y significativo (W de Kendall $=0,864, p<0,001$ ) con una reducción progresiva en las mediciones de 125 [intervalo de confianza (IC) al 95\%: 122,75 a 134,00 inicial vs. 120 (118,75 a 127,75) final].

Este resultado se confirmó mediante mediciones de ultrasonido en las que el edema medio del tejido celular subcutáneo disminuyó en $1,73 \mathrm{~cm}$ (Prueba de Friedman $p=0,000$ ). La temperatura del área tomada antes y después del tratamiento se incrementó en un promedio de $1,4{ }^{\circ} \mathrm{C}$. Evidentemente, es debido a la mejora de la circulación distal y no a un proceso de infección. Estas diferencias son estadísticamente significativas (Wilcoxon $p=0,001$ ) (fig. 2).

Los pacientes que terminaron el proceso de cicatrización fueron 6 , con una tasa de cicatrización porcentual del $60 \%$. En la figura 3 se observa la evolución clínica de la úlcera de un paciente después del tratamiento con radiofrecuencia, con mejoría evidente en la lesión. 

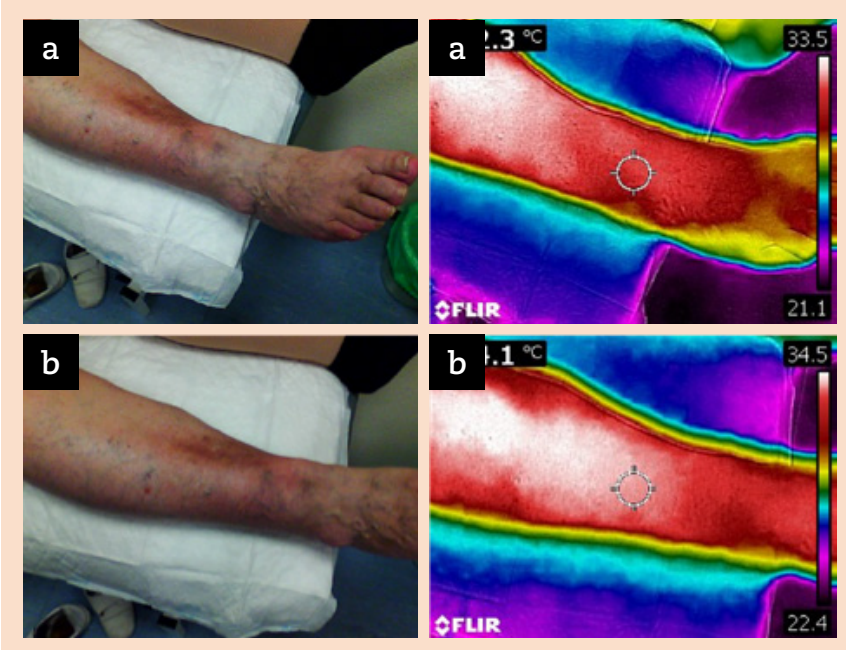

Figura 2. Los cambios de temperatura se observan antes (a) y después (b) del tratamiento.

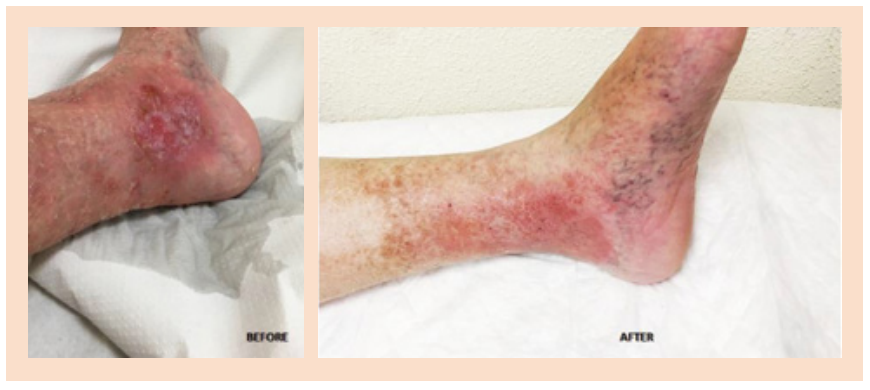

Figura 3. Evolución clínica de la úlcera antes y después del tratamiento con radiofrecuencia.

El dolor se redujo de manera significativa a lo largo del tratamiento $\left[\chi^{2}(9)=261,24, p<0,001\right]$ con un tamańo de efecto grande y significativo (W de Kendall $=0,64, p<0,001$ ), observándose una reducción progresiva en las mediciones de 7 (IC95\%: 4,75 a 7) inicial vs. 3 (2-4) final. Asimismo, en la Escala PUSH (Pressure Ulcer Scale for Healing) se encontraron diferencias significativas a lo largo del tratamiento $\left[\chi^{2}(9)=284,16\right.$, $p<0,001]$ y un tamaño de efecto grande y significativo (W de Kenda$11=0,789, p<0,001)$ con una reducción progresiva en las mediciones de 10,5 (IC 95\%: 7,5, 14,25) inicial vs. $4(4,6)$ ] final (fig. 4).

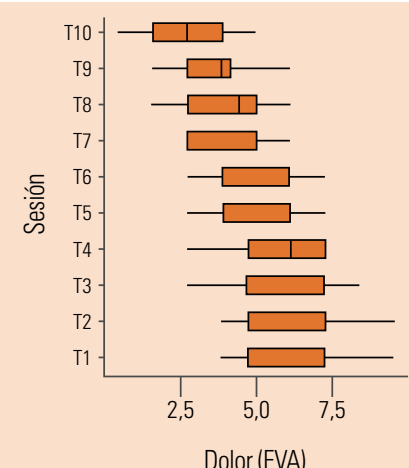

Dolor (EVA)

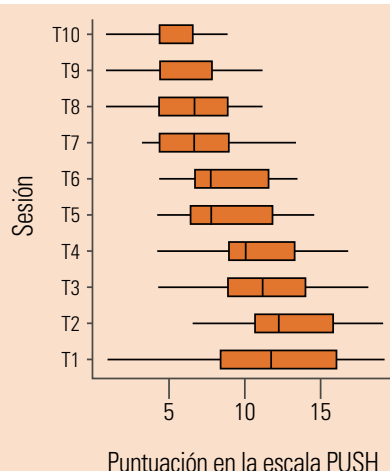

Puntuación en la escala PUSH
Figura 4. Evolución clínica de la úlcera antes y después del tratamiento con radiofrecuencia.

T: momento de las mediciones.

\section{$\searrow$ DISCUSIÓN}

Los resultados presentados muestran los beneficios del uso de la radiofrecuencia en el tratamiento de úlceras tanto en pacientes diabéticos como no diabéticos. Una característica importante es que no es invasivo, no es doloroso, es bien tolerado y los resultados son evidentes: las mejoras de la lesión y subjetivamente el aumento de la calidad de vida de estos pacientes.

En nuestro estudio, en las lesiones se produjeron cambios significativos en la proporción de pacientes con úlcera al principio y al final del estudio, con una reducción en el número de pacientes que la presentaban y un tamaño de efecto grande y significativo. Estos resultados concuerdan con el estudio de George et al. ${ }^{9}$ en el que introdujeron un enfoque terapéutico que utiliza la radioterapia para estimular endógenamente la producción de factores de crecimiento e incitar la mitosis en el lecho de la herida. En estudios in vitro, los fibroblastos primarios humanos y las células epiteliales se trataron con PRFE durante varios periodos y a varias dosis, con proliferación celular evaluada cuantitativamente por recuento directo y análisis espectrofotométrico 24 horas después del tratamiento. Los resultados se compararon con los controles tratados con suero. Los investigadores encontraron un aumento significativo de la proliferación frente al control después de un tratamiento de PRFE de 30 minutos $(p<0,001)$.

Además, sus resultados indicaron que el tratamiento con PRFE induce la producción de factor de crecimiento y estimula la replicación celular a través de una vía intracelular mediada por calcio. Los resultados indican que el tratamiento con PRFE puede ser de valor en el proceso reparador de heridas crónicas. Para probar esta hipótesis, las frecuencias que se utilizan en la radiofrecuencia se aplicaron a pacientes con úlceras crónicas en los miembros inferiores en una zona alejada del sitio de la lesión. Los efectos clínicos inmediatos que se monitorizaron, con resultados idénticos a este estudio, son los que describe la terapia efectiva en pacientes seleccionados con úlceras arteriales y venosas crónicas ${ }^{35-38}$.

Las lesiones de extremidades inferiores se pueden considerar como procesos inflamatorios desregulados producidos por un suministro inadecuado de sangre, anoxia tisular, edema, muerte celular e infección, entre otros factores ${ }^{15}$. Estos cambios alteran la interacción entre los componentes estructurales de los tejidos afectados y entre estos y las células inmunes de una manera que impide la cicatrización de las heridas. Las hipótesis sobre la fisiopatología de las ulceraciones de miembros inferiores se centran en efectos locales inducidos por alteraciones hemodinámicas. Hay tratamientos en la actualidad centrados en el alivio de estos cambios locales que incluyen medidas preventivas hemodinámicas, vendajes para lesiones de larga evolución y tórpidas, tratamientos tópicos y reparación quirúrgica o endovascular ${ }^{20-26}$.

Así, comparativamente con nuestros hallazgos, se produjeron cambios significativos en la proporción de pacientes con edema al principio y al final del estudio. Nos encontramos con una respuesta clínica buena, en la disminución del edema, medimos con eco-Doppler, y se hallaron diferencias significativas a lo largo del tratamiento y un tamaño de efecto grande y significativo con una reducción progresiva en las mediciones. Se ha demostrado que la radiofrecuencia puede tener un efecto sobre los diferentes componentes del mecanismo del proceso de cicatrización. En particular, se refiere a la aplicación de bajas frecuencias y efectos no térmicos. Se ha planteado la hipótesis de que la radiofrecuencia puede influir en la señalización del óxido nítrico, la modulación de los perfiles de citoquinas, la expresión de los factores de crecimiento, la migración celular y la proliferación, y la regulación de la proteína quinasa activada por mitógenos/quinasa regulada por señales extracelulares ${ }^{39,40}$. La aplicación de la radiofrecuencia para la cicatrización de heridas puede 
proporcionar efectos antiinflamatorios junto con la mejora del proceso de reepitelización y mejorar sus efectos en la cicatrización de heridas a través de la regeneración tisular ${ }^{41}$.

\section{Perspectivas futuras}

El empleo de la radiofrecuencia en medicina regenerativa abre una nueva vía para el tratamiento de diversas enfermedades. Debido a su naturaleza no invasiva, su uso tiene ventajas evidentes en comparación con los métodos químicos, biológicos y físicos actuales de regeneración de tejidos y curación de heridas. Teniendo en cuenta el gran potencial clínico de la radiofrecuencia, podemos esperar un aumento en las nuevas técnicas para la regeneración de tejidos y la cicatrización de heridas en una perspectiva cercana. Dicha estrategia permite combinar esta técnica con diversas modalidades químicas, físicas y biológicas para proporcionar bioefectos sinérgicos deseados y una mayor eficacia del tratamiento.

\section{$\vee$ CONCLUSIONES}

La mayor rapidez en la cicatrización de la herida presentada puede explicarse por el efecto antiinflamatorio causado por los cambios en los sistemas de coagulación y anticoagulación, la mejora de la microcirculación y la excreción hormonal. Juntos, contribuyen al aumento de la reactividad inmunológica.

La influencia del campo magnético en el sistema microcirculatorio puede explicar el hecho a menudo citado de que los campos magnéticos tienen efectos antiedematosos, analgésicos y antiinflamatorios, que es una de las razones de su amplia aplicación en la cirugía.

Después de la finalización del tratamiento de radiofrecuencia, parece que puede reducir el largo proceso de cicatrización de las lesiones de evolución tórpida, y nos encontramos con unas diferencias significativas, con un tamańo de efecto pequeño y no significativo, con una reducción progresiva en las mediciones y con una energía absorbida donde hay diferencias significativas a lo largo del tratamiento.

De todas formas, hay que seguir investigando la radiofrecuencia en las heridas, para crear mayor evidencia científica y acercarse a los resultados clínicos

\section{Conflicto de intereses}

Los autores hacen constar que no se ha recibido ningún tipo de financiación (pública o privada) para la realización del proyecto. Así mismo, niegan tener interés económico o personal alguno en los productos que puedan estar descritos en el documento.

\section{У BIBLIOGRAFÍA}

1. Zenevicz IL, Moriguchi Y, Madureira VSF. A religiosidade no processo de viver envelhecendo. Rev esc enferm USP. 2013:47(2):433-9.

2. Lazarus G, Valle MF, Malas M, Oazi U, Maruthur NM, Doggett D, et al. Chronic venous leg ulcer treatment: future research needs. Wound Rep Reg. 2014;22(1):34-42.

3. Fiebig A, Krusche P, Wolf A, Krawczak M, Timm B, Nikolaus $S$, et al. Heritability of chronic venous disease. Hum Genet. 2010;127(6):669-74.

4. Formentini M, Fernandes LP. Factors that influence healing of chronic venous leg ulcers: a retrospective cohort. An Bras Dermatol. 2014;89(3):414-22.

5. Glanz M, Klawansky S, Chalmers T. Biofeedback therapy in stroke: a review. J R Soc Med. 1997:90:33-9.

6. Pelham F, Keith M, Smith A, Williams DV, Powell G. Pressure ulcer prevalence and cost in the U.S. Population. J Am Med Dir Assoc. 2007;8(3):B2.

7. Hirshberg J, Rees RS, Marchant B, Dean S. Osteomyelitis related to pressure ulcers: the cost of neglect. Adv Skin Wound Care. 2000;13(1):25-9.

8. Allman RM. Epidemiology of pressure sores in different populations. Decubitus. 1989:2:30-3

9. George FR, Lukas RJ, Moffett J, Ritz MC. In-vitro mechanisms of cell proliferation induction: a novel bioactive treatment for accelerating wound healing. Wounds. 2002;14:107-15.

10. Mustoe TA, Cooter RD, Gold MH. International clinical recommendations on scar management. Plast Reconstr Surg. 2002:110:560-71.

11. Levenson SM, Geever EF, Crowley LV, Oates JF, 3rd, Berard CW, Rosen $H$. The healing of rat skin wounds. Ann Surg. 1965:161:293-308.

12. Roten SV, Bhat S, Bhawan J. Elastic fibers in scar tissue. J Cutan Pathol. 1996;23:37-42

13. Waldorf H, Fewkes J. Wound healing. Adv Dermatol 1995:10:77-96.

14. Werner S, Grose R. Regulation of wound healing by growth factors and cytokines. Physiol Rev. 2003:83:835-70.

15. Mani R, Falanga V, Shearman CP, Sandaman D. Chronic wound healing. Clinical measurement and basic science. New York: WB Saunders; 1999
16. Bollinger A, Leu AJ, Hofmann U, Franzeck UK. Microvascular changes in venous disease: an update. Angiology. 1997;48:27-32.

17. Browse NL, Burnard KG. The cause of venous ulceration. Lancet. 1982:2(8292):243-5.

18. Cheatle TR, Sarin S, Coleridge Smith PD, Scurr JH. The pathogenesis of skin damage in venous disease: a review. Eur J Vasc Surg. 1991:5:115-23

19. Coleridge Smith PD, Thomas P, Scurr JH, Dormandy JA. Causes of venous ulceration: a new hypothesis. Br Med J. 1988;296:1726-7.

20. Coleridge Smith PD. The microcirculation in venous hypertension. Cardiovasc Res. 1996;32:789-95.

21. Falanga V, Eaglstein WH. The trap hypothesis of venous ulceration. Lancet. 1993;341:1006-8.

22. Second European Consensus Document on Chronic Critical Leg Ischemia. Circulation. 1991;84(Suppl 4):1-25.

23. González de la Torre H, Mosquera Fernández A, Quintana Lorenzo ML, Perdomo Pérez E, Quintana Montesdeoca MP. Clasificaciones de lesiones en pie diabético: Un problema no resuelto. Gerokomos [Internet]. 2012 Jun [citado 7 de enero de 2020]; 23(2):75-87. Disponible en: http://scielo.isciii.es/scielo.php?script=sci arttext\&pid=S1134-928X2012000200006\&lng=es. http://dx.doi.org/10.4321/S1134-928X2012000200006.

24. Ieran $M$, Zaffuto $M$, Bagnacani M, Annovi M, Moratti A, Cadossi R. Effect of low frequency pulsing electromagnetic fields on skin ulcers of venous origin in humans: a double-blind study. J Orthopedic Res. 1990;8:276-82.

25. Stiller MJ, Pak GH, Shupack JL, Taller S, Kenny C, Lorrie J. A portable pulsed electromagnetic field (PEMF) device to enhance healing of recalcitrant venous ulcers: a double blind, placebocontrolled clinical trial. Br J Dermatol. 1992;127:147-54.

26. Lawrence WT. Physiology of the acute wound. Clin Plast Surg. 1998;25:321-40.

27. Levin M. Bioelectric mechanisms in regeneration: unique aspects and future perspectives. Semin Cell Dev Biol. 2009;20:543-56

28. Nuccitelli R. A role for endogenous electric fields in wound healing. Curr Top Dev Biol. 2003:58:1-26

29. Maklebust J, Rodeheaver G, Bartolucci A, Franz RA, Sussman C, Ferrell BA, et al. Pressure Ulcer Scale for Healing:
Derivation and Validation of the PUSH Tool. Adv Wound Care. 1997;10(5):96-101.

30. Chapman CR, Casey KL, Dubner R, Foley KM, Gracely RH, Reading AE. Pain measurement: an overview. Pain. 1985;22:1-31.

31. Ho K, Spence J, Murphy MF. Review of pain-measurement tools. Ann Emerg Med. 1996;27:427-32

32. Meehan DA, McRae ME, Rourke DA, Eisenring C, Imperial FA. Analgesic administration, pain intensity, and patient satisfaction in cardiac surgical patients. Am J Crit Care. 1995;4:435-42.

33. Terai $T$, Yukioka $H$, Asada A. Pain evaluation in the intensive care unit: observer-reported faces scale compared with self-reported visual analog scale. Reg Anesth Pain Med. 1998;23:147-51.

34. Reips U, Funke F. Interval level measurement with visual analogue scales in Internet-based research: VAS Generator. Behav Res Methods. 2008 40: 699-704.

35. Cañedo-Dorantes L, García-Cantú R, Barrera R, Méndez-Ramírez I. Navarro VH, Serrano G. Healing of chronic arterial and venous leg ulcers through systemic effects of electromagnetic fields [corrected]. Arch Med Res. 2002; 33(3):281-9.

36. Matos MA, Cicerone MT. Alternating current electric field effects on neural stem cell viability and differentiation. Biotechnol Prog. 2010;26:664-70.

37. Pesce M, Patruno A, Speranza L, Reale M. Extremely low frequency electromagnetic field and wound healing: implication of cytokines as biological mediators. Eur Cytokine Netw. 2013;24:1-10.

38. Costin GE, Birlea SA, Norris DA. Trends in wound repair: cellular and molecular basis of regenerative therapy using electro-magnetic fields. Curr Mol Med. 2012;12:14-26

39. Pilla AA. Non-thermal electromagnetic fields: from first messenger to therapeutic applications. Electromagn Biol Med. 2013:32:123-36.

40. Sheikh AQ, Taghian T, Hemingway B, Cho H, Kogan AB, Narmo-neva DA. Regulation of endothelial MAPK/ERK signaling and capillary morphogenesis by low-amplitude electric field. J R Soc Interface. 2013;10:20120548.

41. Sunkari VG, Aranovitch B, Portwood N, Nikoshkov A. Effects of a low-intensity electromagnetic field on fibroblast migration and proliferation. Electromagn Biol Med. 2011;30:80-5. 